\title{
Tabaquismo y género: conocimiento sobre enfermedades tabaco- dependientes en la mujer, entre usuarias hospitalizadas en hospitales públicos de Montevideo 2017.
}

\author{
Smoking and gender: knowledge about tobacco-dependent diseases in \\ women, among users hospitalized in public hospitals in Montevideo 2017. \\ Tabagismo e gênero: conhecimento sobre as doenças dependentes do \\ tabaco em mulheres, entre usuários hospitalizados em hospitais públicos \\ em Montevidéu 2017.
}

Antonella Pippo Internista. Asistente de Clínica

Médica.

Laura Llambí Internista, Doctora en Ciencias Médicas. Profesora Agregada de Clínica Médica. Responsable Unidad

Tabaquismo.

Santiago Fontao Estudiante Medicina.

Juan Andrés Albistur Estudiante Medicina.

Juan Carlos Areán Estudiante Medicina.

Nicolás Olmedo Estudiante Medicina.

Ismael Melamed Estudiante Medicina.

Mauricio Minacapilli Residente de Medicina Interna.

Katherin Monzón Estudiante Medicina.

Mary Barros Licenciada en Psicología. Asistente Dpto. de Psicología Médica.

Carolina Parodi Internista. Asistente de Clínica Médica
Resumen: Introducción. El consumo de tabaco es considerado por la Organización Mundial de la Salud la primear causa de muerte evitable a nivel mundial. En Uruguay, $22,2 \%$ de los adultos son fumadores, siendo mayor la prevalencia en mujeres que en varones en el rango etario entre 15 y 18 año. Así mismo, durante el embarazo, hay una frecuencia de tabaquismo del $9,8 \%$ en las pacientes que se atienden en instituciones privadas, y del $26,3 \%$ en instituciones públicas. Se han descripto un conjunto de mitos y desconocimientos que actúan como barreras a la cesación tabáquica en las poblaciones más vulnerables. Objetivo. Determinar el grado de conocimiento sobre enfermedades tabaco-dependientes en general y propias de la mujer, en pacientes hospitalizadas en servicios de ginecología-obstetricia de hospitales públicos de Montevideo, Uruguay en el periodo comprendido entre julio - agosto de 2017. Materiales y métodos. Se realizó un estudio prospectivo, de tipo observacional descriptivo, recolectando datos a través de formularios preestablecidos. Se incluyeron todas las pacientes mayores de 18 años ingresadas en los hospitales públicos seleccionados, que accedieron participar en el estudio. Resultados. Se incluyeron 196 participantes en el estudio con una media de edad de 35,38 años. Un 59\% de la población tenía estudios secundarios incompletos, mientras que el $17 \%$ habían completado solamente primaria. La prevalencia de tabaquismo fue de $30,6 \%$. Las pacientes vinculan el tabaquismo a malos resultados obstétricos en el $96,4 \%$. Solo un $50 \%$ relacionó el tabaquismo al cáncer de cuello uterino, $47,4 \%$ a la infertilidad y $56,1 \%$ a la osteoporosis, mientras que un $86,2 \%$ lo reconoce como factor en el envejecimiento cutáneo. En general, no hubo diferencias significativas de conocimiento entre tabaquistas y no tabaquistas. De entre las participantes, 34,2\% consideraron que fumar tabaco de armar, es menos nocivo que el cigarrillo. Se encontró que una minoría de la población recibió consejería adecuada para el cese durante la internación. Conclusiones. Las pacientes desconocen en parte el vínculo entre el consumo de tabaco y problemas de salud propios de la mujer. Se requiere mayor difusión entre pacientes y equipos de salud de estas áreas, de información y estrategias de consejería, así como derivación para la cesación tabáquica

Palabras clave: tabaquismo, mujer, Uruguay. 
Abstract: Background. Tobacco use is considered as the leading cause of preventable death worldwide by the World Health Organization. In Uruguay, $22.2 \%$ of adults are current smokers. Between 15 and 18 year-old- people prevalence of tobacco use is greater among women. Likewise, during pregnancy, current use stands in $9.8 \%$ in private institutions, and reaches $26,3 \%$ in women assisted in public healthcare services. A combination of myths and lack of awareness had been described as barriers to tobacco cessation in vulnerable populations. Objective. To determine the level of knowledge of tobacco-related diseases among hospitalized patients in public gynecological-obstetric services from Montevideo, Uruguay, during July-August 2017. Materials and methods. Descriptive observational study, obtaining data by means of a survey composed by multiple choice and open-ended questions. Results. 196 patients where included in the study, with a mean of age of 35.38 years. $59 \%$ of the population had incomplete secondary school studies, while $17 \%$ had only completed primary. Tobacco use had a prevalence of $30,6 \%$. Patients related tobacco use to adverse pregnancy results in a $96,4 \%$ of cases. Only $50 \%$ of the population related tobacco use to cervix cancer, $47.4 \%$ to infertility and $56.1 \%$ to osteoporosis. However $86.2 \%$ recognize it as a factor to accelerate skin aging. Generally, there were no significant differences of knowledge between smokers and no smokers. Participants considered chopped tobacco less harmful than cigarettes in $34.2 \%$ of cases. It was found that only a minority of the population received counselling for cessation during hospitalization. Conclusions. Patients are not aware of the link between tobacco use and health problems concerning women. Greater diffusion between patients and health care professionals is required to achieve better results as well as proper referral to cessation clinics.

Key words: tabaquism, women, Uruguay.

Resumo: Introdução. O consumo de tabaco é considerado pela Organização Mundial de Saúde a primeira causa de morte evitável em todo o mundo. No Uruguai, 22,2\% dos adultos são fumantes, sendo a prevalência maior nas mulheres do que nos homens na faixa etária entre 15 e 18 anos. Além disso, durante a gravidez, há uma frequência de tabagismo de 9,8\% em pacientes que frequentam instituições privadas e $26,3 \%$ em instituições públicas. Foi descrito um conjunto de mitos e ignorâncias que atuam como barreiras à cessação do tabagismo nas populações mais vulneráveis. Objetivo. Determinar o grau de conhecimento sobre as doenças dependentes do tabaco em geral e das mulheres, em pacientes internados em serviços de ginecologia obstétrica de hospitais públicos em Montevidéu, Uruguai, no período de julho a agosto de 2017. Materiais e métodos. Foi realizado um estudo descritivo observacional prospectivo, coletando dados através de formulários pré-estabelecidos. Todos os pacientes com idade superior a 18 anos admitidos nos hospitais públicos selecionados, que concordaram em participar do estudo, foram incluídos. Resultados. Incluímos 196 participantes no estudo com uma idade média de 35,38 anos. $59 \%$ da população possuía ensino médio incompleto, enquanto $17 \%$ completaram apenas o primário. A prevalência de tabagismo foi de $30,6 \%$. Pacientes associaram o tabagismo a desfechos obstétricos ruins em $96,4 \%$. Apenas $50 \%$ relacionaram o tabagismo ao câncer do colo do útero, $47,4 \%$ à infertilidade e $56,1 \%$ à osteoporose, enquanto $86,2 \%$ o reconheceram como fator de envelhecimento da pele. Em geral, não houve diferenças significativas no conhecimento entre fumantes e não fumantes. Entre os participantes, 34,2\% consideraram o tabaco como menos prejudicial do que o cigarro. Verificou-se que uma minoria da população recebeu aconselhamento adequado para a cessação durante a hospitalização. Conclusões. Os pacientes desconhecem parcialmente a ligação entre o uso do tabaco e os problemas de saúde da mulher. Maior divulgação é necessária entre os pacientes e equipes de saúde nessas áreas, estratégias de informação e aconselhamento, bem como encaminhamento para cessação do tabagismo.

Palavras-chave: Fumar, mulher, Uruguai.

Recibido: 18/04/2018 - Aceptado: 01/06/2018 


\section{Introducción}

El consumo de tabaco es considerado por la Organización Mundial de la Salud (OMS) como la primera causa de muerte evitable a nivel mundial y su control y tratamiento se encuentran dentro de las acciones trazadas para alcanzar las metas de salud de los Objetivos de Desarrollo Sostenible. ${ }^{(1)}$

En Uruguay la prevalencia del consumo ha descendido en forma sostenida desde 2006 hasta la actualidad como resultado dela política de control de tabaco. Actualmente $22,2 \%$ de los adultos son fumadores y $9,2 \%$ de losjóvenes, siendo en éstos levemente superior en mujeres que en varones. ${ }^{(2,3)}$

No obstante, el descenso no ha sido homogéneo en los diversos estratos socioeconómicos. El consumo de tabaco en el quintil de menores ingresos alcanza el 29,9\% en Uruguay mientras que es de $17,2 \%$ en el quintil de mayores ingresos económicos. (2) Consistentemente, en las mujeres embarazadas que se asisten en instituciones privadas en Uruguay se detecta un $9.8 \%$ de consumo de tabaco durante el embarazo, mientras que alcanza un $26,3 \%$ entre aquellas que se asisten en instituciones públicas. ${ }^{(4,5)}$ Existen estudios previos realizados en embarazadas de sectores vulnerables que muestran la existencia de mitos o desconocimiento que actúan barreras a la motivación para dejar de fumar. ${ }^{(6)}$

A nivel global, el tabaquismo en las mujeres era muy infrecuente en la primera mitad del siglo XX. Cambios sociológicos, como la incorporación al mundo laboral, los movimientos de igualdad, y el constituirse en un blanco específico del mercado para la industria tabacalera propiciaron la introducción de las mujeres al consumo de tabaco. ${ }^{(7,8)}$

En consecuencia, la incidencia de enfermedades cardiovasculares y respiratoriasyalgunos cánceres se ha incrementadosignificativamente en dicho género.

Existen además, ciertas patologías causadas o favorecidas por el consumo de tabacoo la exposición al humo de segunda mano (HSM) ${ }^{(7)}$ específicas de la mujer que son menos conocidas por la población general. Entre estas se encuentran por ejemplo, la osteoporosis, la infertilidad, la menopausia precoz, neoplasia de cuello uterino y otras vinculadas a la esfera obstétrica sobre las cuales hay menos difusión entre las usuarias. ${ }^{(8)}$

El objetivo del presente estudio fue determinar el conocimiento que tienen las usuarias hospitalizadas en servicios de ginecología y obstetricia de dos instituciones públicas deMontevideo sobreenfermedades específicas de la mujer relacionadas con el consumo de tabaco.

\section{Material y Métodos}

\section{a) Diseño}

Se realizó un estudio prospectivo, de tipo observacional descriptivo.

\section{b) Población}

Se incluyeron de forma sistemática todas las pacientes de sexo femenino mayores a 18 años ingresadas a sala de internación ginecológica u obstétrica en las Clínicas Ginecotocológicas "A" y "C" del Centro HospitalarioPereira Rossell y "B" del Hospital de Clínicas Dr. Manuel Quintela, durante el período comprendido entre el $1^{\circ}$ de julio y el 31 de agosto de 2017.

Fueron criterios de exclusión el no consentimiento a participar o poseer cualquier tipo de discapacidad que les impidiese consentir de forma autónoma.

\section{c) Variables de estudio}

Las variables de estudio fueron: edad, nivel educativo, información con respecto a las enfermedades tabaco-dependientes en general y propias de la mujer, condición degestante, estatus tabáquico. El estatus tabáquico se categorizó de acuerdo a definiciones universales en: fumadoras diarias (al menos un cigarrillo al día todos los días); fumadoras ocasionales(al menos 1 cigarrillo en los últimos 6 meses pero menos que uno al día); ex fumadoras (aquellas que habiendo sido fumadoras no consumen hace por lo menos 6 meses); en abstinencia (aquellas que habiendo sido fumadoras no consumen desde hace menos de 6 meses). Salvo edad, que fue considerada como una variable numérica continua, las variables a investigar fueron consideradas como cualitativas dicotómicas o politómicas. 


\section{d) Recolección de los datos}

Se entrevistaron en forma sistemática todas las usuarias que ingresaron en las salas de hospitalización de los servicios participantes que cumplían los criterios de inclusión y no poseían criterios de exclusión. Para la recolección de los datos se utilizó un formulario en papel, administrado y registrado por el entrevistador.

\section{e) Análisis estadístico}

Se presentan los datos en frecuencias absolutas y relativas y en medias y desvío estándar. Se utilizó test de $\ 2$ para comparar proporciones.Se consideró estadísticamente significativo un valor de $p$ menor a 0,05. Los datos se procesaron mediante Microsoft Excel e IBM SPSS v19.

\section{f) Aspectos éticos}

El estudio adhirió a las disposiciones del decreto 379/008 del Ministerio de Salud Pública referente a la investigación con seres humanos y a la Declaración de Helsinki versión 2000. Las participantes recibieron información verbal y escrita previa a su ingreso al estudio y firmaron consentimiento informado. El proyecto fue aprobado por los Comités de ética del Hospital de Clínicas y Centro Hospitalario Pereira Rossell.

\section{Resultados}

Se incluyeron196 pacientes con un rango etario comprendido entre los 18 y 77 años siendo la media de la edad 35,38 años y desvío estándar (DE) 16,25. Con respecto al nivel educativo $12 \%$ poseían primaria incompleta, $17 \%$ primaria completa y la mayoría (59\%) habían cursado estudios secundarios incompletos. Del total, 55 (28,10\%) estaban embarazadas.

Las variables relacionadas con su tabaquismo se describen en la tabla 1. Se destaca que $30,6 \%$ eran fumadoras actualesincluyendo fumadoras diarias y ocasionales.

\begin{tabular}{|l|l|l|}
\multicolumn{1}{c|}{ Estatus tabáquico } & \multicolumn{1}{c|}{ Frecuencia absoluta } & $32,1 \%$ \\
\hline No fumadora & 63 & $6,1 \%$ \\
\hline Fumadora pasiva & 12 & $13,8 \%$ \\
\hline En abstinencia & 27 & $17,3 \%$ \\
\hline Ex fumadora & 34 & $6,6 \%$ \\
\hline Actual ocasional & 13 & $24 \%$ \\
\hline Actual diaria & 47 & $100 \%$ \\
\hline Total & 196 & \\
\hline
\end{tabular}

Entre las pacientes que reportaron ser fumadoras, ex fumadoras o encontrarse en abstinencia $(n=121), 38,8 \%$ refirió haber recibido consejería vinculada a la cesación tabáquica durante su internación. Entre las que habían logrado cesar su consumo en alguna oportunidad refirieron haberlo logrado sin apoyo terapéutico el $87 \%$, mientras que el resto había recibido algún tipo tratamiento farmacológico específico o apoyo grupal.

Las pacientes conocían las repercusiones del tabaquismo en el embarazo aún en consumos de menos de 5 cigarrillos al día en $96,4 \%$ de los casos. Mencionaban en forma espontánea (mediante pregunta abierta) que el fumar se relacionaba con enfermedades cardiovasculares, respiratorias, neoplásicas y otras, con las frecuencias mostradas en la Gráfica 1. Un 34,2\% de las pacientes consideraba que consumir tabaco armado era menos nocivo que fumar cigarrillos.

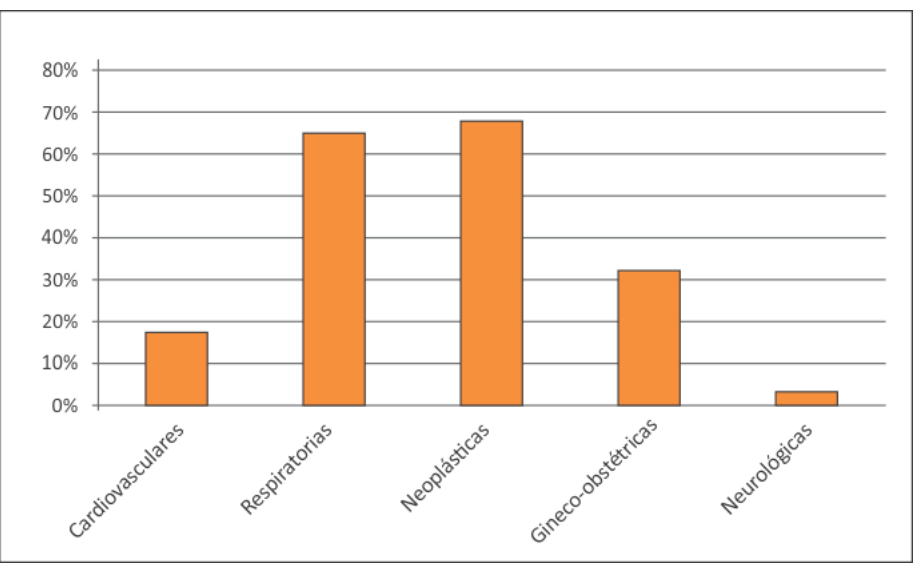


Cuando se investigó el conocimiento de las usuarias acerca del vínculo entre el tabaquismo y otras enfermedades propias del género o con afectación diferencial en la mujer se encontró que solo $50 \%$ conocían la asociación entre consumo de tabaco y cáncer de cuello uterino (CCU) y dos de cada tres mujeres consideraba que el consumo de tabaco estaba implicado en el desarrollo del cáncer de mama.

Enfermedades tales como la osteoporosis, infertilidad y envejecimiento cutáneo fueron reconocidas como asociadas al tabaquismo en $56,1 \%, 47,4 \%$ y $86,2 \%$ respectivamente. En la Gráfica 2 se muestran estas y otras afecciones mencionadas por las usuarias como vinculadas al consumo de tabaco.

Gráfica 2: Porcentaje de paciente que vincula el consumo de tabaco con enfermedades tabaco-dependientes, propias

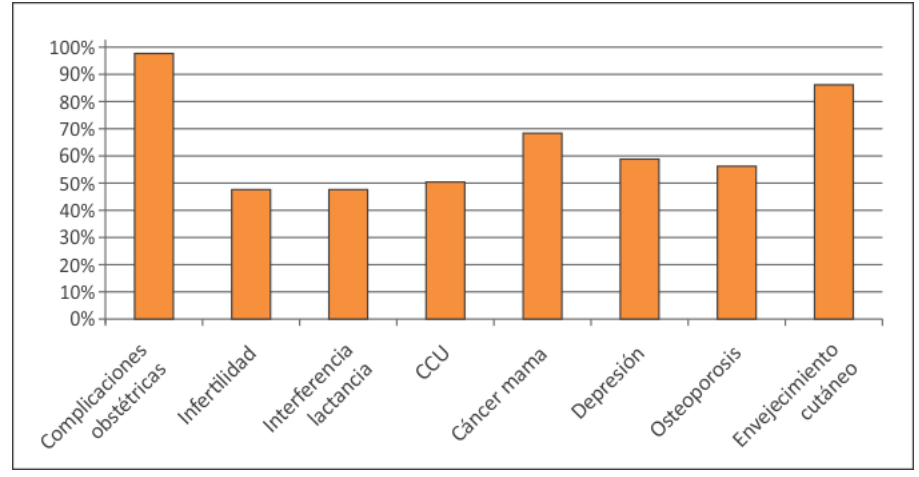

CCU: cáncer de cuello uterino

Se categorizaron a las encuestadas en dos grupos según estatus tabáquico, uno constituido por las no fumadorasy otro por pacientes fumadoras, en abstinencia y ex fumadoras para investigar diferencias en los conocimientos sobre enfermedades tabaco-dependientes entre ambos grupos. No se encontraron diferencias significativas entre ambos grupos, salvo en lo que refiere al conocimiento sobre el vínculo entre el tabaco y la patología respiratoria no oncológica. Las pacientes no fumadoras mencionaban al tabaquismo como factor asociado a padecer enfermedades respiratorias no oncológicas en $77,3 \%$, mientras que las fumadoras lo hacían en $57 \%$ de los casos. $(p=0,005)$

\section{Discusión}

El porcentaje de fumadoras en la población estudiada fue de $30,6 \%$, mayor a la media poblacional. Este hallazgo es consistente con los datos nacionales que muestran mayores niveles de prevalencia de consumo de tabaco en los sectores más vulnerables desde el punto de vista socio-económico. Es de destacar que aun encontrándose en una ventana de oportunidad para lograr la abstinencia tabáquica como lo es la hospitalización muchas continúan el consumo (9). Entre las que lograron cesar en el momento de ser entrevistadas o lo habían logrado en oportunidades previas, se destaca la subutilización de los servicios y programas específicos de cesación de tabaquismo. Los programas de cesación integrales como los que están disponibles en el sistema de salud uruguayo, que cuentan con apoyo conductual y farmacológico logran hasta triplicarla posibilidad de lograr y mantener con éxito la cesación tabáquica por lo que su subutilización debería ser abordada y corregida. ${ }^{(10)}$

Se observó que menos de la mitad (38,8\%) recibieron durante la internación, algún nivel de consejería o derivación para abandonar el tabaco. Este hallazgo es similar al hallado a nivel poblacional en Uruguay, cuando se investigó el porcentaje de consejo breve que reciben los fumadores que asisten por cualquier motivo a una consulta de salud. ${ }^{(11)}$ Las posibles explicaciones son multifactoriales, y están relacionadas con el factor tiempo en la consulta, formación específica para dar la consejería, pocas expectativas acerca de la efectividad del consejo breve de cesación, entre otros. $(6,12-14)$

El consejo breve para dejar de fumar es una herramienta probadamente efectiva, que incrementa los intentos de cesación tabáquica y las tasas de abstinencia. El requerir solamente dos a tres minutos y poder ser efectuada por todo el equipo de salud con mínimo entrenamiento y en todos los niveles de atención lo convierten en una estrategia importante a nivel poblacional. (15)

Interesa destacar que entre las usuarias existe la percepción del riesgo de fumar en el embarazo, y en una amplísima mayoría no se encontró el concepto erróneo previo de un umbral de seguridad de 5 cigarrillos/día. De acuerdo a la evidencia actual, el fumar en el embarazo es la primera causa evitable de malos resultados obstétricos-perinatales, recomendándose el cese completo en forma precoz como nivel de recomendación I. (16-22) En contrapartida, existe desconocimiento sobre la igual nocividad del tabaco armado respecto al cigarrillo. 
Con referencia al conocimiento de las mujeres acerca de las enfermedades tabacodependientes, se destaca el desconocimiento sobre algunas de ellas específicas de la mujer o con particular impacto en el sexo femenino. Estas afecciones son también menos reconocidas por los equipos de salud como asociadas al tabaquismo y por lo tanto menos incluidas en los temas de consejería anti tabáquica. Dentro de las enfermedades del área de salud mental, la depresión es significativamente más frecuente en fumadoras y en mujeres expuestas al humo de segunda mano de acuerdo a la evidencia del estudio de cohortes de Copenhague y al estudio de Kim y colaboradores. $(23,24)$

Poco más de la mitad de las pacientes reconocía la asociación entre tabaquismo y osteoporosis. El consumo de tabaco está fuertemente asociado a una menor densidad mineral ósea, fractura osteoporótica y fractura de cualquier causa. $(25,26)$

Similar proporción de pacientes conocían la asociación entre tabaquismo y neoplasias de la mujer. El consumo de tabaco es un reconocido factor de riesgo para el cáncer de cuello uterino, así como para el desarrollo de lesiones precursoras, carcinoma in situ y neoplasia cervical intraepitelial grado 3. ${ }^{(27)}$ Su papel como co-factor de la infección por Virus del Papiloma Humano ha sido más recientemente estudiado y demostrado.(28)

Entre las sustancias tóxicas conocidas del humo de tabaco, algunos de ellos han sido identificadas carcinogénicos mamarios. Sin embargo la asociación entre tabaquismo y desarrollo de cáncer de mama es controvertida aún. Existen estudios que muestran asociación entre el tabaquismo intenso por más de 30 años y el riesgo de padecer cáncer de mama en la posmenopausia. (29-31) Un metaanálisis realizado por Duan y colaboradores concluyó que las fumadoras muestran un aumento de la mortalidad específica por cáncer de mama y que en cambio las ex fumadoras tienen una mortalidad específica por cáncer de mama muy reducida. (32) Otros estudios que también apoyan el rol del tabaquismo en el desarrollo de cáncer de mama subrayan como factores influyentes la edad temprana de inicio del consumo, sobretodo antes de un primer embarazo. ${ }^{(33)}$

Controversialmente otros autores reportan una nula asociación entre el estatus tabáquico, su duración e intensidad con el riesgo de incidencia del cáncer de mama. ${ }^{(34)}$

En nuestro estudio, a excepción del conocimiento sobre patología respiratoria no neoplásica vinculada al consumo de tabaco, no se encontraron diferencias significativas entre los conocimientos sobre enfermedades tabaco dependientes de las fumadoras con respecto a las no fumadoras. Si bien informar a las usuarias sobre los daños a la salud que causa el tabaquismo y personalizar el consejo breve de acuerdo a la patología que presenten forma parte de la estrategia de cesación, existen otros factores tales como la motivación, la oportunidad, el uso de tratamiento farmacológico específico que inciden en el logro de la misma. 


\section{Conclusiones}

La prevalencia de tabaquismo entre las usuarias hospitalizadas en servicios de ginecología y obstetricia de hospitales públicos es elevada. En la mayoría de los casos no se realiza la consejería de cesación tabáquica apropiada. Las pacientesdesconocen en parte el vínculo entre el consumo de tabaco y problemas de salud propios de la mujer. Se requiere mayor difusión entre pacientes y equipos de salud de estas áreas, de información y estrategias de consejería, así como derivación a unidades especializadas encesación tabáquica

\section{Agradecimientos}

Agradecemos a todas las participantes que accedieron a ingresar a nuestro estudio, así también al personal del Centro Hospitalario Pereira Rossell y Hospital de Clínicas. También deseamos agradecer alas cátedras de Métodos Cuantitativos y Bioética de la Facultad de Medicina por el apoyo brindado. 


\section{Bibliografía}

1- United Nations. UN Sustainable Development Goals [Internet]. 2015. [consultado 17/3/2018] Disponible en: http://www.un.org/sustainabledevelopment/health/

2- Uruguay. Instituto Nacional de Estadística. Encuesta Continua de Hogares [Internet]. 2014.[consultado 17/3/2018]Disponible en: http://www.ine.gub.uy/encuesta-continua-de-hogares1

3- Uruguay. Junta Nacional de Drogas. $6^{\circ}$ Encuesta Nacional sobre Consumo de Drogas en Estudiantes de Enseñanza Media.Observatorio Uruguayo de Drogas: Montevideo: 2014.

4- Uruguay. Instituto Nacional de Estadística; Ministerio de Salud Pública. Salud, nutrición y desarrollo en la primera infancia en Uruguay: primeros resultados de la Encuesta de Nutrición, desarrollo infantil y Salud [Internet]. 2015. [consultado 17/3/2018].Disponible en: http://www.ine.gub.uy/ documents/10181/35704/SALUD,+NUTRICI\%C3\%93N+Y+DESARROLLO+EN+LA+PRIMERA+IN FANCIA+EN+URUGUAY+PRIMEROS+RESULTADOS+DE+LA+ENDIS/7be3f504-ebb9-4427-bb5dcb4d9f242a7b

5- Althabe F, Aleman A, Berrueta M, Morello P, Gibbons L, Colomar M, et al. A multifaceted strategy to implement brief smoking cessation counselling during antenatal care in Argentina and Uruguay: a cluster randomized trial. Nicotine Tob Res. 2015;1-11.

6- Colomar M, Tong VT, Morello P, Farr SL, Lawsin C, Dietz PM, et al. Barriers and Promoters of an Evidenced-Based Smoking Cessation Counseling During Prenatal Care in Argentina and Uruguay. Matern Child Health J. 2015; 19(7):1481-9.

7- Centers for Disease Control and Prevention (US). The Health Consequences of Smoking for Women. A Report of the Surgeon General. Public Health Service, Office of the Assistant Secretary for Health, Atlanta,GA; 2001.

8- World Health Organization. Gender, women and the tobacco epidemic [Internet]. WHO: Geneva; 2010 [consultado 17/3/2018]. Disponible en: http://www.who.int/tobacco/publlications/gender/women_tob_ epidemic/en

9- Pippo A, Llambi L, Parodi C, Barros M, Nuñez V, Silveira A, et al. Missed Opportunities of Tobacco Cessation Interventions in Hospitalized Patients. J Fam Med. 2016;3(9):1089.

10- Uruguay. Ministerio de Salud Pública. Guía Nacional para el Abordaje del Tabaquismo. Montevideo: MSP; 2009.

11- Uruguay. Ministerio de Salud Pública. Global Adult Tobacco Survey [Internet]. WHO; 2009. [consultado 17/3/2018]. Disponible en: http://www.paho.org/uru/index.php?option=com_ content\&view $=$ article\&id $=111 \& \mid$ temid $=1$

12- Blumenthal D. Barriers to the Provision of Smoking Cessation Services Reported by Clinicians in Underserved Communities. J Am Board Fam Med. 2007;20:272-9.

13- Cooke M, Mattick R, Walsh R. Differential uptake of a smoking cessation programme disseminated to doctors and midwives in antenatal clinics. Addiction. 2001;96:495-505.

14- Llambí L, Barros M, Parodi C, Blanco L, Esteves E, Cora M, et al. Conocimientos, creencias practicas y actitudes con respecto al consumo de tabaco de estudiantes de Medicina de primer año en Uruguay , 2009. Rev Med Urug. 2012;28(1):4-12.

15- Stead LF, Buitrago D, Preciado N, Sanchez G, Hartmann-Boyce J, Lancaster T. Physician advice for smoking cessation. Cochrane database Syst Rev [Internet]. 2013;5:CD000165. [consultado 17/3/2018] Disponible en: http://www.ncbi.nlm.nih.gov/pubmed/23728631

16- World Health Organization. WHO recommendations for the prevention and management of tobacco use and second-hand smoke exposure in pregnancy [Internet]. 2013.[consultado 17/3/2018]Disponible en: http://www.who.int/tobacco/publications/pregnancy/guidelinestobaccosmokeexposure/en/

17- Berrueta M, Morello P, Alemán A, Tong VT, Johnson C, Dietz PM, et al. Smoking Patterns and Receipt of Cessation Services Among Pregnant Women in Argentina and Uruguay. Nicotine Tob Res 2015; 18 (5):1116-25

18- Llambi L, Aleman A, Colomar M, Morello P, Sosa L, Arcos J, et al. Weight Gain in Women after Smoking Cessation in Pregnancy. J Smok Cessat. 2017;12(3):131-8.

19- Herman HG, Miremberg H, Nini N, Feit H, Schreiber L, Bar J, et al. The effects of maternal smoking on pregnancy outcome and placental histopathology lesions. Reprod Toxicol. 2016;65:24-8.

20- Balte P, Karmaus W, Roberts G, Kurukulaaratchy R, Mitchell F, Arshad H. Relationship between birth weight, maternal smoking during pregnancy and childhood and adolescent lung function: A path analysis. Respir Med. 2016;121:13-20. 
21- Leonardi-Bee J, Smyth A, Britton J, Coleman T. Environmental tobacco smoke and fetal health: systematic review and meta-analysis. Arch Dis Child Fetal Neonatal Ed. 2008 Sep;93(5):F351-61.

22- Iñiguez C, Ballester F, Amorós R, Murcia M, Plana A, Rebagliato M. Active and passive smoking during pregnancy and ultrasound measures of fetal growth in a cohort of pregnant women. J Epidemiol Community Health. 2012;66(6):563-70.

23- Talbott JA. Tobacco smoking as a risk factor for major depressive disorder: population-based study. Year B Psychiatry. 2010;163-4.

24- Kim NH, Kim HC, Lee JY, Lee J-M, Suh I. Association between environmental tobacco smoke and depression among Korean women. BMJ Open. 2015;5(6):e007131.

25- Thorin MH, Wihlborg A, Åkesson K, Gerdhem P. Smoking, smoking cessation, and fracture risk in elderly women followed for 10 years. Osteoporos Int. 2016;27(1):249-55.

26- Yoon V, Maalouf NM, Sakhaee K. The effects of smoking on bone metabolism. Osteoporos Int. 2012;23(8):2081-92.

27- Roura E, Castellsagué X, Pawlita M, Travier N, Waterboer T, Margall N, et al. Smoking as a major risk factor for cervical cancer and pre-cancer: Results from the EPIC cohort. Int J cancer. 2014;135(2):45366.

28- Jensen KE, Schmiedel S, Frederiksen K, Norrild B, Iftner T, Kjær SK. Risk for cervical intraepithelial neoplasia grade 3 or worse in relation to smoking among women with persistent human papillomavirus infection. Cancer Epidemiol Prev. 2012;21(11):1949-55.

29- Connor AE, Baumgartner KB, Baumgartner RN, Pinkston CM, Boone SD, John EM, et al. Cigarette smoking and breast cancer risk in Hispanic and non-Hispanic white women: The breast cancer health disparities study. J Women's Health (Larchmt). 2016;25(3):299-310.

30- Gaudet MM, Gapstur SM, Sun J, Diver WR, Hannan LM, Thun MJ. Active smoking and breast cancer risk: original cohort data and meta-analysis. J Natl Cancer Inst. Oxford University Press US 2013;105(8):515-25.

31- Xue F, Willett WC, Rosner BA, Hankinson SE, Michels KB. Cigarette smoking and the incidence of breast cancer. Arch Intern Med. 2011;171(2):125-33.

32- Duan W, Li S, Meng X, Sun Y, Jia C. Smoking and survival of breast cancer patients: A meta-analysis of cohort studies. The Breast. 2017;33:117-24.

33- Bjerkaas E, Parajuli R, Weiderpass E, Engeland A, Maskarinec G, Selmer R, et al. Smoking duration before first childbirth: an emerging risk factor for breast cancer? Results from 302,865 Norwegian women. Cancer Causes Control. 2013;24(7):1347-56.

34- Catsburg C, Kirsh VA, Soskolne CL, Kreiger N, Rohan TE. Active cigarette smoking and the risk of breast cancer: a cohort study. Cancer Epidemiol. 2014;38(4):376-81. 